\title{
The Difficulties and Optimization Mechanism of Big Data Application in SMEs
}

\author{
Yi Liu *, Pingxiu LI \\ School of Business Administration, Neusoft Institute Guangdong \\ ${ }^{*}$ Corresponding author. Email: liuyi@nuit.edu.cn
}

\begin{abstract}
In the era of digital economy, big data has become a strategic resource, and big data application capabilities have also become a strategic capability, which can effectively assist SMEs in precision marketing, enhancing corporate competitiveness, and achieving quality and efficiency improvements. However, the application of big data for SMEs is facing a series of difficulties such as insufficient understanding and lack of big data thinking, not enough investment and incomplete big data infrastructure, irregular management and low quality of basic big data, incomplete introduction and lack of big data talents. For this reason, it is necessary to raise awareness and establish big data thinking, increase investment and improve big data infrastructure, standardize management and improve the quality of basic big data, and pay equal attention to talent introduction and education, and strengthen the construction of big data talents.
\end{abstract}

Keywords: Big data, SMEs, Application, Difficulties, Optimization Mechanism.

\section{INTRODUCTION}

In the era of digital economy, advanced information technologies such as mobile Internet, cloud computing, big data, artificial intelligence, and the Internet of Things appear continuously, and further penetrate into all areas of society, resulting in many new application scenarios and profoundly changing our social style, lifestyle and work style. Big data has gradually become a strategic resource. In this regard, large companies, especially leading companies, have long been aware of the power of big data, and have vigorously deployed related fields, actively invested capital and talents, and carried out big data-related construction and applications to realize the value and improvement of big data. However, in contrast, small and medium-sized enterprises(hereinafter SMEs) are at a disadvantage in terms of big data hardware, technology, and talent reserves which caused by both objective constraints and subjective constraints. For this reason, it is necessary to correctly understand the significance of SMEs' big data application, analyze the difficulties they face, and propose corresponding solutions in order to improve the performance, value and resource utilization of big data application, and further enhance the competitiveness of SMEs[1].

\section{BIG DATA APPLICATION PROCESS}

The big data application process usually includes four links: big data acquisition, big data processing, big data analysis, and big data presentation. It is worth noting that, based on the continuity and spiral of organizational activities and decision-making, for the operation of enterprises, big data application is more often not a one-way process, but a cyclical process of acquisition-processing-analysis-presentation.

\subsection{Big data acquisition}

To apply big data and play the value of big data, enterprises first need to have basic big data, that is, the acquisition of big data. The sources of enterprise big data usually include the following: first, the data that has been or is being generated in the internal history and current activities of the enterprise; second, the data that has been or is being generated in the history and current activities of the external organizations or individuals; third, the data that generated by the connection and interaction between enterprises and external organizations or individuals. Enterprises can obtain big data by self-built databases, data collection from economic and social activities, and data purchases from third parties. The quality of big data acquired by 
enterprises, especially the matching degree between data and demand, has an important impact on the efficiency and effectiveness of big data application.

\subsection{Big data processing}

Enterprises must clean or organize the data acquired from inside and outside the organization before analyzing and presenting the data if they want to realize the value of big data application[2]. The purpose of big data processing is to accumulate high-quality data for subsequent big data analysis and presentation, especially to integrate, clean, and extract basic big data to ensure the availability of data in subsequent big data applications. For big data application, a considerable amount of time and energy is spent on big data processing, and the quality of big data processing is directly related to the efficiency and effectiveness of big data applications.

\subsection{Big data analysis}

Big data analysis is the core part of big data application. The most important thing is to look at the essence through the phenomenon by big data analysis, that is, to discover and even predict changes in consumer demand and preference, grasp customer needs in a timely manner, and improve customer satisfaction; to find out the problems in current corporate activities, improve work processes, optimize resource allocation, etc. The most common directions of big data analysis include data mining and predictive analysis. The former represents the analysis of the past and the present, and the latter represents the analysis of the future. The application of big data analysis has attracted the attention of many scholars in the past ten years. Research shows that big data analysis is a key factor driving the success of organizations, and big data analysis has a significant positive impact on organizational performance[3].

\subsection{Big data presentation}

The results of big data analysis usually need to be presented directly and concisely in a certain form (such as charts, videos, brief reports, etc.), rather than in complex and difficult to understand tables or text descriptions. Big data presentation is also called big data visualization. Its tools are generally divided into three categories: interactive visualization tools, configuration visualization tools, and programming visualization tools. The presentation of big data is very important to reflect the value of big data application in itself, but only a means. For example, when a beverage company analyzes and predicts consumer preferences for beverage categories, the core conclusions of the analysis may be displayed using only a pie chart. Of course, with the diversification of business and popularization of data visualization smart tools, the presentation of big data is more often in the form of smart dashboards, which requires enterprise employees to have professional skills.

\section{SIGNIFICANCE OF BIG DATA APPLICATION IN SMES}

\subsection{Big data application capability has become a strategic capability}

The With the increasing attention of big data and the increasing development of its application value, big data has gradually become a strategic resource. Big data has 4 major characteristics (ie 4V), namely: Volume (large volume), Velocity (high speed), Variety (diversity), Value (low value density). Big data application refers to the process of collecting, storing, and analyzing data with a huge amount, scattered sources, and various formats, and discovering new knowledge, creating new value, and improving new capabilities from it. Big data application capability is the ability of completing the process. Therefore, the ability to correctly understand big data, effectively apply big data, and tap its value is becoming a strategic capability of enterprises.

\subsection{Big data application is an effective means for SMEs to achieve precision marketing}

The application and management activities of big data such as big data collection and big data analysis in the operation of SMEs can help companies accurately understand and master the consumer needs, preferences and consumption cycles of customers, so as to achieve precise marketing and agile marketing as well as promotion. In this process, it is possible for camponies to enhance the customer's consumption experience, establish a strong customer stickiness, and improve marketing performance[4], which is of great benefit to stabilize the passenger flow and establish long-term cooperation, and is helpful to the sustainable development of SMEs.

\subsection{Big data application is a realistic choice for SMEs to improve quality and efficiency}

SMEs can use big data to support decision-making, promote business innovation, promote business process optimization and business model innovation, and then achieve cost reduction, quality improvement, and efficiency increase[5]. For example, cross-border ecommerce of SMEs can timely and accurately grasp changes in consumer demand through big data collection and analysis in various business links, and reflect and integrate consumer demand changes in a short period of time in design, production and sales by its flexible decision-making mechanism and operating mechanism, so as to transform from "sales center 
theory" or "profit center theory" to "customer center theory", effectively reducing operating costs, improving operating efficiency, and achieving cost reduction, quality improvement and efficiency.

\subsection{Big data application of is an important path for SMEs to enhance their competitiveness}

Big data is not only a technological change, but also an economic change. Therefore, it can be said that big data is becoming a new driving force for economic and social development. In the new stage of digitization, intelligence, and information development, big data has become the focus of competition in various countries around the world, and this is also true for industries and enterprises. Big data is changing the comprehensive competitiveness of industries and enterprises and reshaping future industry development and competitive landscape. In terms of traditional competition factors such as costs, products, channels, etc., SMEs do not have an advantage, or even are at a relatively obvious disadvantage. It is difficult for SMEs to compete with large enterprises, especially leading enterprises. But big data and its application have changed this situation to a certain extent. For example, small and medium-sized clothing customization companies can use big data to analyze customized customer portraits, precise customers, fashion trends, etc., launch smart recommendations and clothing matching, and conduct online and offline integrated marketing and so on, so as to enhance the competitiveness of enterprises and increase the competitive advantage.4. Difficulties of big data application in SMEs.

\section{DIFFICULTIES OF BIG DATA APPLICATION IN SMES}

\subsection{Insufficient understanding and lack of big data thinking}

There seems to be a natural conflict between the "big" data and the "small" and medium-sized enterprises. Therefore, most of the SMEs, from managers to employees, have insufficient understanding of big data and believe that only big companies have the big data, need the big data, and can apply the big data. In fact, with the development of advanced technologies, big data and its applications have penetrated into all walks of life and integrated into the development of industries and enterprises. The lack of big data thinking and understanding has caused some SMEs hardly to learn, digest, absorb and innovate quickly in the rapidly changing market, which lead to a difficulty to accurately respond and adjust to the market regardless of their products and services, or production, sales, promotion and other operational activities, or even the overall planning and business model design of organizations, thus missing some development opportunities.

\subsection{Not enough investment and incomplete big data infrastructure}

Big data applications have certain requirements for basic hardware settings and equipment. The equipment required for big data storage, calculation, and analysis and its performance are relatively high. Traditional computer hardware and supporting software cannot meet the needs of big data-related applications. Due to the lack of professional knowledge and overall planning, some SMEs do not have the corresponding funds and manpower to invest in the construction and application of big data, or even if they have sufficient funds and manpower, it is difficult to carry out the scientific and reasonable deployment and construction of big data. Ultimately, the utilization rate of funds is low. After the waste of manpower and material resources, the value of big data application cannot be highlighted within the enterprise, so its construction is difficult to receive further support. In general, the current big data investment of SMEs is not enough, and the infrastructure is not complete.

\subsection{Irregular management and low quality of basic big data}

Due to the lack of big data thinking and big data concepts, there is less conscious accumulation and management of big data in the process of enterprise management and operation. To better manage and apply big data, firstly SMEs need to have basic big data, otherwise it will be "nothing to cook." Secondly, the quality of basic big data greatly affect the application value of big data. High-quality data should have completeness, accuracy, validity, consistency, and availability. Whether the data is complete, accurate, and effective, whether the data from different sources is consistent, and whether the data is available when needed, all affect the efficiency of big data application. From a practical point of view, a considerable number of SMEs have failed to accumulate basic data that meets the above characteristics through standardized and effective management, resulting in the inability to efficiently apply big data and further reflect its value.

\subsection{Incomplete introduction and lack of big data talents}

The current lack of big data professionals is an important reason why it is difficult for SMEs to promote big data management and application. Today, with the increasingly widespread use of big data, the talent gap for big data has further increased. Big data management and application talents are no longer traditional technical talents, but comprehensive and compound talents who can master big data technology and are familiar with business and business analysis. Big data application is often closely related to cloud computing, 
Internet of Things, artificial intelligence, etc., and will use these technologies more as important means of big data collection, big data mining, and big data analysis to further improve the efficiency and quality of mining and processing and meet the user needs in the future. With the further integration of big data with the Internet, cloud computing, artificial intelligence, etc., companies in all industries will need more compound talents in the future. At present, there is a shortage of big data talents to meet this demand in China, and there is a large imbalance between market demand and talent supply which is increasing day by day. According to a research report by CCID, the core talent gap for China's big data is expected to reach 2.3 million in 2025. For SMEs, there are fewer big data talents either self-cultivated or imported from outside, and the lack of talents directly restricts the big data application and the improvement of the benefits.

\section{THE OPTIMIZATION MECHANISM OF BIG DATA APPLICATION IN SMES}

\subsection{Raise awareness and build big data thinking}

It is necessary for SMEs to improve their understanding of big data and establish a comprehensive thinking about big data. Through the establishment of big data-related systems, full-staff training, creation of typical cases, honor recognition, publicity and promotion, etc., the popularization of big data knowledge and education and training are carried out, so that members of all levels and types of organizations can realize the importance of big data application and experience the positive impact that big data application may have in improving work efficiency, reducing work burdens, improving organizational performance, and avoiding backward development, etc., and internally accept and recognize big data application, establish big data thinking, and further consciously, organized, planned and actively participate in the enterprise's big data application and construction and other organizational activities, thus improve the overall awareness and application level of organization members.

\subsection{Increase investment and improve big data infrastructure}

Based on the importance and necessity of infrastructure for the effective big data application, SMEs should attach importance to increasing investment in big data-related equipment and facilities to lay a solid foundation. At the same time, it should be combined with the actual financial situation of the company, avoid blindly pursuing the most advanced and expensive equipment to highlight the importance of big data application, but consider adopting a variety of ways other than directly purchasing products. The "Action Program for Promoting the Development of Big Data" clearly supports companies in developing third-party data analysis and discovery services, technology outsourcing services, and knowledge process outsourcing services based on big data, and encourages large and medium-sized enterprises to coordinate development and collaborative innovation. Therefore, SMEs can adopt flexible and diverse forms such as third-party leasing and purchasing professional services or using Cloud deployement creatively to achieve comprehensive coverage and improvement of big data infrastructure[6].

\subsection{Standardize management and improve the quality of basic big data}

In the course of business operations, SMEs should focus on improving the standardization of management, especially strengthening the management of data, improving the overall data quality, and paying attention to the completeness, accuracy, validity, consistency, and availability of data. In the daily operation of the enterprise, a large amount of data is accumulated, and at the same time, more data is continuously generated outside the enterprise. On the one hand, SMEs must continue to carry out standardized management, so that the current and future data itself is standardized and usable. On the other hand, companies should formulate data standards for a large amount of internal and external data, and continue to improve data quality and data governance, use professional technical means and professional technical personnel to continuously discover data problems, and continuously improve and enhance data quality, for example monitor data quality by statistical process control to avoid sudden deterioration of data quality, and negative impact on data or even business caused by abnormal values, and provide enterprises with more comprehensive and accurate data, in order to lay a solid foundation for the efficiency of big data application.

\subsection{Pay equal attention to introduction and cultivation of talents, and strengthen the construction of big data talents}

Talent is a key factor in the development of an enterprise. The introduction of talent can promote the transformation and upgrading of the enterprise, the enhancement of competitiveness, and the improvement of technological innovation capabilities. SMEs should pay equal attention to the introduction and cultivation, and do a good job in the reserve and use of big data professionals. At present, the competition for big data talents in all walks of life is becoming more and more intense, especially the Internet and technology companies are making every effort to increase their commitment to fields related to big data (such as cloud 
computing, Internet of Things, machine learning, artificial intelligence, etc.), intensify the introduction of talents, and provide generous treatment conditions to attract top big data talents. In this competitive situation, it is difficult for SMEs to compete in a short period of time. For this reason, SMEs can appropriately bypass this competition track and adopt more feasible and operational solutions to attract big data talents. On the one hand, when recruiting personnel, SMEs should consciously choose industry talents with certain big data knowledge and skills who already possess both big data skills and industry experience. On the other hand, SMEs should conduct big data-related skills training and promotion for existing personnel.

University-Business Cooperation (UBC) is an important mechanism for advancing interests of business, universities and societies[7], therefore schoolenterprise cooperation with universities and vocational colleges and customized talent training are also key considerations for SMEs in the cultivation of big data talents. The AUCP program launched by Alibaba Cloud in 2015 has successively cooperated with more than 40 universities in China, including Zhejiang University, Fudan University, Shanghai Jiaotong University, South China University of Technology, etc., to set up cloud computing and data science majors, cultivate big data professionals. In recent years, the "e-commerce customized classes" and "big data experimental classes" that have achieved good results are typical examples of school-enterprise cooperation in cultivating outstanding industry talents. SMEs can refer to these practices to formulate big data talent cultivation plans, and use school-enterprise cooperation to realize the refined training and customized employment of big data talents, so as to obtain compound big data talents who possess big data knowledge and skills and understand the industry and even have certain industry experience at a lower cost and in a shorter period of time.

\section{CONCLUSION}

The importance of big data application has been paid more and more attention, and has even become a consensus, and the quality of big data application is also playing an increasingly important role[8]. In the digital economy era, if SMEs want to continuously improve their competitiveness, it is necessary for them to build the entire process of high-quality big data application from awareness to behavior, from basic investment to process control, so as to realize the value of big data application.

\section{ACKNOWLEDGMENTS}

This research was financially supported by the Young Innovative Talent Project/ Characteristic Innovative Talent Project of Guangdong Provincial Department of Education (Grant NO. 2020WQNCX081
/2020WTSCX112) and the Scientific Research and Innovation Team Building Project of Neusoft Institute Guangdong (Grant NO. 2020XKYTD01).

\section{REFERENCES}

[1] U. Utsav and S. Geeta. MRS-DP: Improving Performance and Resource Utilization of Big Data Applications with Deadlines and Priorities. Big data, 2020, vol.8, no.4, pp. 323-331.

[2] K. Rahul, R. K. Banyal and P. Goswami. Analysis and processing aspects of data in big data applications. Journal of Discrete Mathematical Sciences and Cryptography, 2020, vol. 23, no. 2, pp. 385-393.

[3] M. Q. Shabbir and S. B. W. Gardezi. Application of big data analytics and organizational performance: the mediating role of knowledge management practices. Journal of Big Data, 2020, vol. 47, no. 7, pp. $1-17$.

[4] G. Shivam; J. Théo; K. Shampy; K. Ajay; K. Eivind. Big data and firm marketing performance: Findings from knowledge-based view[J]. Technological Forecasting \& Social Change, 2021, vol. 171, in press.

[5] A. I. Aljumah, M. T. Nuseir and M. M. Alam. Organizational performance and capabilities to analyze big data: do the ambidexterity and business value of big data analytics matter?[J]. Business Process Management Journal, 2021, vol. 27, no. 4, pp. 1088-1107.

[6] S. Karimian-Aliabadi, D. Ardagna, R. EntezariMaleki, E. Gianniti and A. Movaghar. Analytical composite performance models for Big Data applications. Journal of Network and Computer Applications, 2019, vol. 42, pp. 63-75.

[7] B. Orazbayeva, C. Plewa, T. Davey and V. G. Muros. The Future of University-Business Cooperation: Research and Practice Priorities[J]. Journal of Engineering and Technology Management, 2019, no. 54, pp. 67-80.

[8] J. Shunhui, L. Qingqiu, C. Wennan, Z. Pengcheng and M. Henry. Quality Assurance Technologies of Big Data Applications: A Systematic Literature Review[J]. Applied Sciences, 2020, vol. 10, no. 22, pp. 8052-8052. 